\title{
New Methods for the One-Electron Problem
}

\author{
O. Krogh Andersen, Stuttgart
}

(Max-Planck Institute for Solid State Research)

EPS Hewlett-Packard Europhysics Prize Winner for 1980. The article contributed by the co-winner, A.R. Miedema was published in the September issue.

One aim of solid state research is to improve our understanding of the chemical and physical properties of materials. Why do atoms combine into solids in the way they do and what is the response of these solids to electromagnetic fields, stress, temperature etc. ? An improved understanding of such questions could be a useful guide in our, so far mostly empirical, search for new and better materials: stronger materials, materials which corrode less, better catalysts, better magnets, better semi- or superconductors, and, materials with properties unknown today. I expect that within the coming decades, many advances will be initiated by approximate electronic structure calculations.

The properties of materials are, at the microscopic level, governed by the behaviour of the electrons because these light particles in their motion almost immediately follow the far heavier nuclei. The basic problem is therefore to calculate the stationary states for the system of electrons moving in the electrostatic field of the fixed nuclei; that is, the electronic structure. At a later stage, the energy of the electronic ground state obtained as a function of the nuclear positions can serve as potential energy for the motion of the nuclei.

The electronic-structure problem for a solid is one of infinitely-many interacting fermions, but for realistic materials we can, as a first step, at most attempt to solve Schrödinger's equation for one electron moving in the mean field of the other electrons plus the field of the nuclei. This mean field is composed of the electrostatic field from the electronic charge cloud, plus corrections for exchange and correlation, and it is usually determined by the following self-consistency procedure. For a supposed total field, $V$, one-electron energies, $E_{i}$, and wavefunctions, $\psi_{i}$, are obtained by solving the one-electron Schrödinger equation (in atomic units) :

$$
\left(-\nabla^{2}+V-E_{j}\right) \psi_{j}=0 \text {. }
$$

The one-electron wavefunctions are then occupied according to the Pauli principle, and a new field is obtained by solving Poisson's equation for the nuclear pointcharges screened by the electronic charge cloud of density:

$n(\mathbf{r})=\Sigma_{\text {jocc. }}\left|\psi_{j}(\mathbf{r})\right|^{2}$,

and by addition of the corrections for exchange and correlation. With this new field, or rather a weighted sum of the old and the new field, the calculation is now repeated and the cycle iterated until the input and output fields are consistent. From the one-electron energies and wavefunctions, the total electronic energy may be estimated, while the one-electron energies themselves, are approximations to the oneelectron excitation energies.

The classical self-consistent field method is the Hartree-Fock which, by definition, neglects correlation. For a solid, the method would not only be laborious because the exchange field depends in a complicated way on the one-electron state in question, but it would also yield too large a separation between occupied and unoccupied one-electron levels, and hence make a subsequent treatment of correlation necessary.

The mean field used in calculations for solids is a much simpler, state-independent potential, $V(r)$, that can be constructed from the electron density alone, and which includes important effects of both exchange and correlation through the use of accurate many-body calculations for a simple model system. The model system is taken to be the so-called jellium, in which the nuclei are smeared out to a uniform background. Taking the contribution from exchange and correlation to the total energy to be $\varepsilon_{x c}(n)$ per electron, in a jellium of density $n$, the contribution in the real system can be expressed as

\section{$\int \varepsilon_{c x}(n(\mathbf{r})) n(\mathbf{r}) d^{3} r$.}

This co-called local approximation to density functional theory works surprisingly well and examples for solids will be given below. Even for isolated atoms and small molecules, the method yields energies of the ground state and low-lying excited states which are more accurate than those obtained with the Hartree-Fock method. So much we have learned during the past ten years, partly with the aid of the oneelectron techniques that I shall describe below. Nevertheless, before self-consistent calculations of total energies became feasible, one-electron calculations with a local exchange potential had been highly successful in describing the band structures of the elemental semiconductors and the Fermi surfaces of the elemental metals.

The motivation therefore existed to devise methods for computing one-electron energies and wavefunctions with the

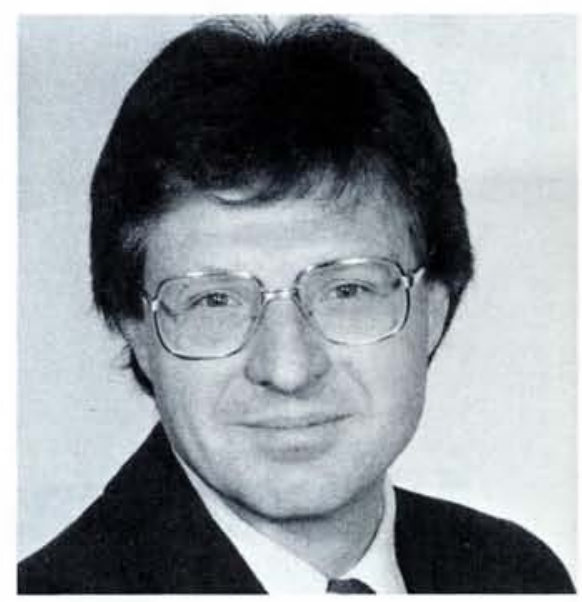

speed and accuracy needed in self-consistent calculations. Such a method should apply to atomic constituents from all parts of the periodic table and potentially be applicable to realistic structures: crystals with many atoms per cell, crystals with surfaces, interfaces and impurities, or even clusters of impurities, amorphous systems, molecules and so on. In addition to being numerically accurate, fast and reliable, such a one-electron method should be physically transparent and thereby have the flexibility to be used at various levels of approximation. It could then form the basis for analytical models which would make the computed results intelligible and hence open the way for further progress.

The new, so-called linear methods of band theory') go some way towards meeting the above requirements and I shall now try to explain the essential ideas behind these methods.

\section{ONE ELECTRON METHODS}

We want to solve Schrödinger's equation (1) for the bound states in a potential, $V(r)$, characteristic for a system of atoms. A part of such a potential is shown in Fig. 1a where, specifically, we show the behaviour near the surface of a crystal, in a plane perpendicular to the surface. The atoms in the surface layer are labelled $S$ and those in the third crystal layer $\mathrm{C}$. For an infinite crystal the potential is of course simpler than this. The energy range of interest to most materials scientists extends from about -2 to 0 Rydberg.

\section{Traditional Methods}

There are two traditional approaches to the one-electron problem, the bound-state and the partial-wave approach. The first approach is used in the linear combination of atomic orbitals (LCAO) method, where the potential is approximated by a superposition of spherically symmetric atomic potentials and the wavefunctions, $\psi_{i}(\mathbf{r})$, are approximated by linear combinations of atomic orbitals, $\Sigma_{\mathbf{R} n / m} a_{i, \mathbf{R} n / m} \chi_{\mathbf{R} n / m}(\mathbf{r}-\mathbf{R})$. Here $\mathbf{R}$ labels the type and position of the atom and $n, I$ and $m$ are principal and angular-momentum quantum numbers. 
The atomic orbital is a product of a spherical harmonic, $Y_{I m}(\hat{P})$, and a bound radial function, $\chi_{R n !}(r)$. The unknown coefficients, $a$, and the one-electron energies, $E$, may then, by use of the variational principle, be obtained as the eigenvectors and the eigenvalues of the algebraic eigenvalue problem

$(H-E O) a=0$,

with the hamiltonian matrix,

$H=\left\langle\chi_{\mathrm{R}^{\prime} n^{\prime} m^{\prime} m^{\prime}}\left|-\nabla^{2}+V\right| \chi_{\mathrm{R} n / m}\right\rangle$,

and the overlap matrix,

$$
O=<_{\mathrm{R}^{\prime} n^{\prime} \mathrm{m}^{\prime}}\left|\chi_{\mathrm{R} n / m}\right\rangle \text {. }
$$

This LCAO method has the advantages of using a local description based on the atomic constituents and of leading to an algebraic eigenvalue problem. Difficulties lie in the choice of a sufficiently small and accurate set of orbitals and in the necessity to compute a large number of integrals involving orbitals and potentials centred at two and three different sites. This is so complicated that it is customary to neglect all but the two-centre integrals between the nearest and next nearest neighbours, and to treat these integrals as adjustable parameters.

In the partial-wave approach, space is divided into regions inside which the potential is averaged in such a way that ble. As an example we show in Fig. $1 \mathrm{~b}$ the so-called muffin-tin average of the potential in Fig. 1a. Inside spheres surrounding the atoms, the muffin-tin average is spherically symmetric, in the vacuum region it only depends on the distance from the surface, and in the remaining, so-called interstitial region it is constant.

For this approximate potential it is now possible to solve Schrödinger's equation exactly in terms of energy-dependent partial-wave expansions, $\Sigma_{L} b_{\mathbf{R} L} \phi_{\mathbf{R} L}(E, r-R)$, in the various regions. Inside a spherical region, $L$ labels the angular momentum, $I m$, and the partial wave is the product of a spherical harmonic and the radial function, $\phi_{R /}(E, r)$, obtained by integrating (numerically) the radial Schrödinger equation at energy $E$ outwards from the centre to the sphere radius. In a vacuum region, $L$ labels and the partial wave is $\exp \left(\mathbf{i K} \cdot \mathbf{r}_{=}\right)$times the solution, $\phi_{\mathrm{RK}}\left(E, r_{\perp}\right)$, obtained by integrating the $r_{\perp}$-dependent Schrödinger equation at energy $E$, inwards from infinity

The one-electron energies, $E_{\mathrm{j}}$, are now those values of $E$ for which $b$-coefficients can be found such that the partial-wave expansions join continuously and differentially together at the boundaries between the regions. The way in which this matching condition is formulated algebraically, differs for the various partial-wave methods, but in general, the result is a set of linear, homogeneous equations

$M(E) b=0$ Schrödinger's equation becomes separathe momentum, $K$, parallel to the surface to the surface.
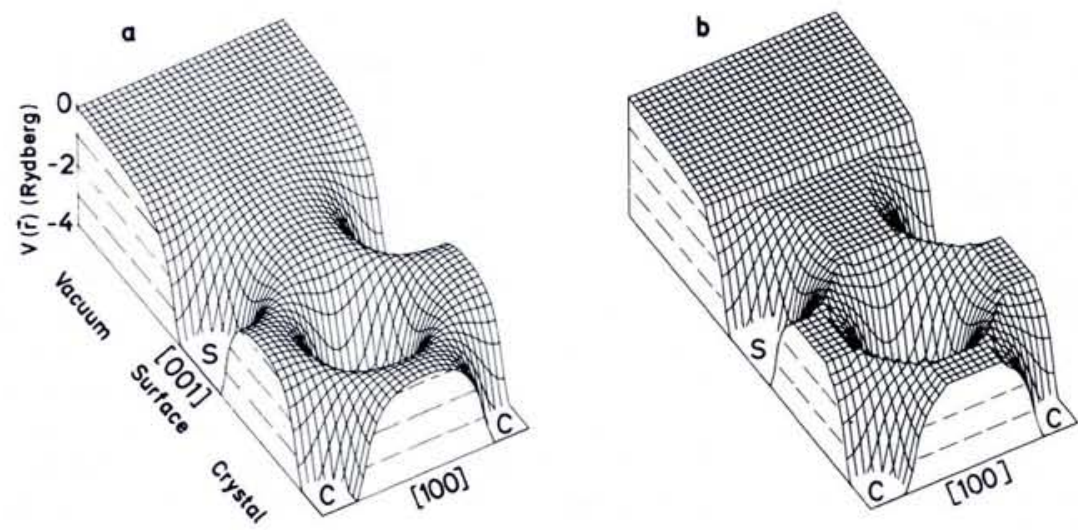

Fig. 1 - a: One-electron potential in a plane perpendicular to the surface of a nickel crystal. $S$ and $C$ denote atoms in, respectively, the surface plane and the third crystal plane below the surface. The potential is self-consistent and was obtained with the linear augmented-plane-wave method.

$b$ : The muffin-tin average of the potential shwon in Fig. 1a: Inside the touching spheres surrounding the atoms, the muffin-tin potential is spherically symmetric, in the vacuum region, it only depends on the distance from the surface plane and in the remaining, interstitial region, it is constant. to the matrix $H$-EO in (2) has a complicated, non-linear energy dependence. As a consequence, the one-electron energies, $E_{i}$, and the corresponding solutions, $b_{j, R L}$, must be found individually by first tracing a root of the determinant of $M$, and then solving the linear equations.

Even for moderate sized matrices this requires orders of magnitude more computation than does the solution of the eigenvalue problem (2). Further disadvantages of the partial-wave methods are that their formalisms are complicated and that perturbations, such as the remaining, nonmuffin-tin part of the potential, are difficult to include in (3) because this condition does not derive from the variational principle for the one-electron hamiltonian. The partial-wave approach does, however, have two distinct advantages: it provides solutions of arbitrary accuracy for the muffin-tin potential, and for close-packed crystals this makes the partial-wave methods far more accurate than any traditional bound-state method. Furthermore the information about the potential enters
(3) only via a few functions of energy, the logarithmic derivative functions. For a spherical region these are essentially the slopes of the radial functions at the sphere boundary, or precisely, $\partial \ln \left|\phi_{R^{\prime} /}(E, r)\right| / \partial \ln r$ for $r=s$.

Among the partial-wave methods are the so-called cellular method of Wigner and Seitz, the augmented-plane-wave (APW) method of Slater and the scattered-wave method of Korringa, Kohn and Rostocker (KKR). Popular bound-state methods are the pseudo-potential and LCAO methods.

\section{Linear Methods}

The linear methods employ the boundstate approach (2), but their basis functions are defined in such a way that, when applied to a muffin-tin potential, they can provide solutions of arbitrary accuracy like the partial-wave methods. The linear methods, moreover, use only logarithmic derivative parameters to describe the potential. In this way the linear methods combine the desirable features of the bound-state methods with those of the with a secular matrix, $M$, which in contrast

\section{0 - 31 July, 1981}

\section{NATO ADVANCED STUDY INSTITUTE}

\section{ELECTRON CORRELATIONS IN SOLIDS}

Spin and charge density waves, density-functional formalism, transport properties of many-electron systems, influence of exchange and correlations on lattice dynamics, local fields, effective interactions in metals, Wigner condensation, quasi-twodimensional electron states, surface plasmons, inelastic electron scattering in metals and plasmon dispersion experiments, plasmons in inhomogeneous many-electron systems, collective excitations in solids, molecules and atoms.

Preliminary list of lecturers:

W. Kohn, Santa Barbara; S. Lundquist, Goteborg; G. Niklasson, Goteborg; A. Overhauser, West-Lafayette; P. Platzman, Murray Hill; G. Priftis, Patras; J. Ruvalds, Charlottesville; S. Schnatterly, Charlottesville; K. Singwi, Evanston; M. Tosi, Trieste; J. Devreese, Antwerp.

Scientific Committee: J.T. Devreese (Chairman), F. Brosens, P. Van Camp.

Information: International Advanced Study Institute

Department of Physics (ESIS-secretariat), University of Antwerp (U.I.A.)

Universiteitsplein 1, B - 2610 WILRIJK 


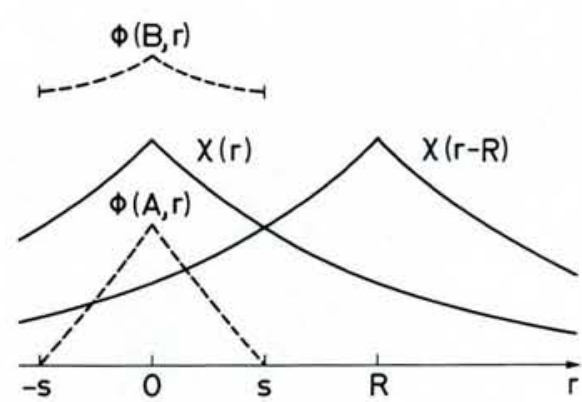

Fig. 2 - Atomic orbitals, $\chi$, and partial waves, $\phi$, for a diatomic molecule (schematic). The atoms are centred at $O$ and $R$, and the radius of the muffin-tin sphere at $O$ is s. Inside the atom at the origin is the bonding state $\chi(r)+\chi(r-R) \cong \phi(B, r)$, and the antibonding state $\chi(r)-\chi(r-R) \cong \phi(A, r)$.

partial-wave methods and they have none of the drawbacks.

In order to explain how the basis functions of the linear methods are defined we consider in Fig. 2 a diatomic molecule, crudely approximated by two muffin-tin spheres, and compare the LCAO description with the partial-wave description of the bonding and antibonding states. These are $\chi(r) \pm \chi(r-R)$ in terms of the energyindependent, overlapping atomic orbitals and they are $\phi(B, r)$ and $\phi(A, r)$ in terms of the energy-dependent partial wave at the atom at the origin. Here $B$ and $A$ are the energies of the bonding and antibonding states. We thus understand that the difference, $\phi(B, r)-\phi(A, r)$, in the LCAO description should be supplied by the tail of the orbital, $\chi(r-R)$, at the other site. In a solid, where a whole band of states exists with energies between $B$ and $A$, the entire energy dependence of the partial wave should be supplied by the tails of the orbitals from the other sites. Now, the linear part of the Taylor series

$\phi(E, r)=\phi(r)+\left(E-E_{v}\right) \dot{\phi}(r)+\ldots$

is well converged between $B$ and $A$ if $E_{v}$ is chosen appropriately, and we shall therefore try to augment (i.e. substitute) the tail

Fig. 3 - Radial d-function for yttrium, $\phi\left(E_{v}, r\right)$, and the energy-deviation function, $\dot{\phi}\left(E_{v}, r\right)$, which is orthogonal to it. The Taylor expansion was chosen around an energy, $E_{\mathrm{v}}$, equal to the centre, $C$, of the $4 d$-band.

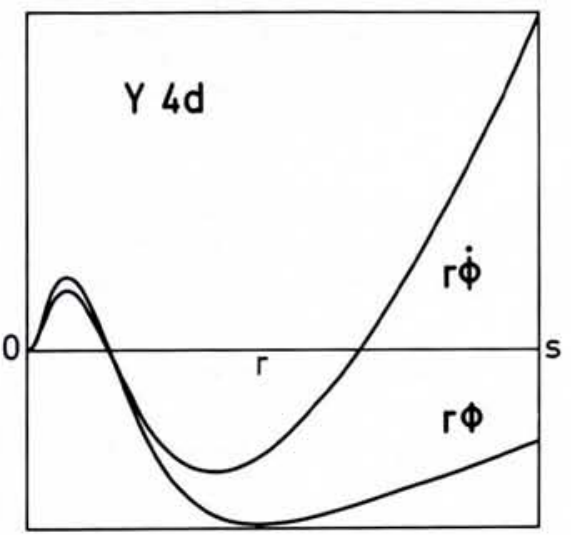

inside the overlapped atom by the energyderivative of the partial wave at $E_{y}$, that is, by $\dot{\phi}$. At the same time we shall try to augment the orbital inside its own sphere by some other linear combination, say, $\phi(r)+\left(C-E_{v}\right) \dot{\phi}(r)$.

This augmentation of the orbital can, in fact, be performed continuously and differentiably because the radial phi and phidot functions have different logarithmic derivatives at the sphere, such that a linear combination of them can substitute any radial function inside the sphere with a continuous and differentiable matching at the sphere. The augmentation can even be performed by a phi-dot function only because this function is not unique; the arbitrary linear combination of phi and phi-dot, $c \phi(r)+d \dot{\phi}(r)$, is the phi-dot function corresponding to the normalisation $\left[d+\left(E-E_{v}\right) c\right] \phi(E, r)$. In view of this nonuniqueness we shall from now on specify the normalisation by $\left\langle\phi^{2}(E)\right\rangle=1$ in the sphere. This means that phi is normalized to unity and that phi-dot is orthogonal to phi in the sphere. An example of such an orthogonal pair is shown in Fig. 3.

From an atomic orbital we thus construct a basis function for a linear method, by expanding the orbital inside each muffin-tin sphere in spherical harmonics times radial functions, and then substitute the latter by appropriate linear combinations of phi and phi-dot. For a geometry like the one shown in Fig. 1b the augmentation should also be performed in the vacuum region. The resulting orbital is everywhere continuous and differentiable and it equals the atomic orbital only in the interstitial region. This augmented atomic orbital is, however, not very practical because its expansion coefficients are complicated and its kinetic energy in the interstitial region is too low for solid-state potentials, but it should be obvious that any smooth set of functions, $\chi_{J}$, which is reasonably complete in the interstitial region, can be augmented as described above and then used as basis functions in a linear method. We may generally write

$$
\begin{aligned}
\chi_{J}(\mathbf{r})= & \chi_{J}^{i}(\mathbf{r})+\sum_{R L} \pi_{J, R L} \phi_{\mathbf{R} L}(\mathbf{r}-\mathbf{R}) \\
& +\Omega_{J, \mathbf{R} L} \phi_{\mathbf{R} L}(\mathbf{r}-\mathbf{R}),
\end{aligned}
$$

where $\chi^{i}$ and phi and phi-dot are supposed to vanish outside, respectively, the interstitial and the appropriate spherical regions. The matrices $\pi$ and $\Omega$ contain the spherical harmonic expansion coefficients and the potential parameters, which are the values of phi and phi-dot and their radial derivatives at the sphere boundary.

It is now a simple matter to write down the hamiltonian and overlap matrices for use in (2). For each sphere and angular momentum we have $\left\langle\phi^{2}\right\rangle=1$ and $\langle\phi \dot{\phi}\rangle=0$. Moreover, the spherical parts of the hamiltonian matrix follow trivially from:

$$
\begin{gathered}
\left(-\nabla^{2}+V-E_{v}\right) \phi=0 \text { and } \\
\left(-\nabla^{2}+V-E_{v}\right) \dot{\phi}=\phi .
\end{gathered}
$$

The ease with which the remaining integral over the interstitial region and the matrix elements of the non-muffin tin part of the potential can be performed, depends on the basis set. For crystals, the set of plane waves is convenient and accurate, and the potential shown in Fig. 1a is, in fact, the result of a self-consistent calculation with the so-called linear augmentplane-wave (LAPW) method. A drawback of the plane-wave set is that it needs $20-50$ plane-waves per atom while the orbital set, that I shall describe in a following section, needs only $\left(t_{\max }+1\right)^{2}=4-16$ orbitals per atom.

If the basis set is overcomplete in the interstitial region, as is for instance the set of plane waves, and if the potential has the muffin-tin form, the only source of error in a linear method is the first-order expansion (4). Consequently the wavefunctions obtained by solving the eigenvalue problem (2) have errors of second and higher order in $E_{i}-E_{v}$ and the eigenvalues, being variational estimates, have errors of fourth and higher order in $E_{j}-E_{\mathrm{v}}$. This means that a linear method can be viewed as a boundstate method which needs only one (arbitrary and non-integer) value, $v$, of the principal quantum number per atom and angular momentum.

In Fig. 4 we show the energy-band structure of palladium metal in the wide range from about 0.5 Rydberg below the Fermi level to 4 Rydberg above. In order to cover this large range, where the narrow $4 \mathrm{~d}$-bands and the wide $5 \mathrm{~s}-, 5 \mathrm{p}$-, $5 \mathrm{~d}$ - and $4 \mathrm{f}$-bands are situated, three calculations with three different values of $E_{v}$ were performed. The good overlap between the three densities of states obtained indicates the smallness of the fourth-order errors.

\section{Atomic Spheres Approximation}

In closely packed solids each atom has 8-12 nearest neighbours and the muffin-tin

Fig. 4 - Calculated density of one-electron states in face-centered-cubic palladium metal. The energy range of over 4 Rydberg, starting at the bottom of the $5 s$-band, was covered by three linear bandstructure calculations using the values of $E_{\mathrm{v}}$ shown. The Fermi level is at 0.5 Rydberg.

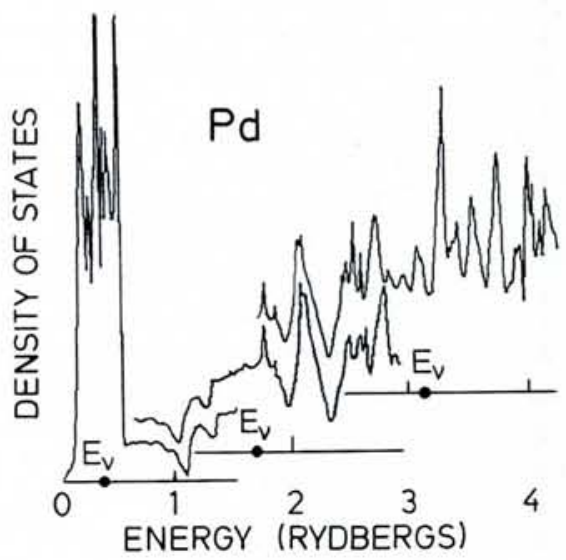


approximation (Fig. 1b) is good so long as the kinetic energy, $E-V$, in the region between the atoms is so low that the corresponding wave-length is much larger than the extent of the interstitial region. Nearly as accurate, and far more convenient, is an approximation in which the interstitial region is "annihilated" through expansion of the muffin-tin spheres (and of the vacuum region) and neglect of the slight overlap. The muffin-tin spheres in this approximation thus become the Wigner-Seitz atomic spheres which are supposed to fill space.

In open structures the interstitial positions often have so high a symmetry that not only the atomic, but also the repulsive interstitial, can be approximated by spherically symmetric potentials and the atomic and interstitial spheres taken together form a close packing. In such cases, the atomic spheres approximation is also a good starting point. We shall now use this approximation to derive an explicit and simple description of the electronic structure.

First we construct that set of orbitals for which the tails are augmented by that phidot function which is orthogonal to phi. In terms of some finite basis set, (5), this new set of orbitals comprises the linear combinations specified by the application of the matrix $\pi^{-1}$, i.e.

$$
\theta_{R L}(\mathbf{r}-\mathbf{R})+\sum_{\mathbf{R}^{\prime} L^{\prime}}, H_{\mathbf{R} L, \mathbf{R}^{\prime} L^{\prime}}, \dot{\phi}_{\mathbf{R}^{\prime} L^{\prime}}\left(\mathbf{r}-\mathbf{R}^{\prime}\right)
$$

It is easily verified that $H$, defined as $\pi^{-1} \Omega$, is in fact the hamiltonian matrix (relative to $E_{v}$ ) in the representation of the new orbitals, (6), and that the overlap matrix is $0=1+\mathrm{H}<\dot{\phi}^{2}>\mathrm{H}$.

Here $\left\langle\dot{\phi}^{2}\right\rangle$ is a matrix diagonal in $\mathbf{R}$ and $L$. Realistic values of the potential parameter $\left\langle\dot{\phi}^{2}\right\rangle^{-1 / 2}$ are several Rydbergs, so that the second term in (7) is small.

We have thus constructed a set of orbitals which are orthogonal to first order in energy. It is furthermore plausible that this set is localised, because the tails must fall off rapidly in order to match the strongly increasing phi-dot function as seen in Fig. 3 . With this set it is now a simple matter to solve Schrödinger's equation. We first diagonalise $H$ obtaining the eigenvectors $b$ and eigenvalues $E_{i}-E_{v}$. The corresponding wavefunctions obtained from (6) are seen to be

$$
\begin{gathered}
\psi_{j}(\mathbf{r})=\Sigma_{\mathbf{R} L} b_{j, \mathbf{R} L}\left[\phi_{\mathrm{RL}}(\mathbf{r}-\mathbf{R})+\right. \\
\left.\left(E_{j}-E_{v}\right) \phi_{R L}(\mathbf{r}-\mathbf{R})\right],
\end{gathered}
$$

in direct analogy with the previously mentioned energy-dependent partial-wave expansion, and correct to first order in energy. The eigenvalues, $E_{i}$, have errors of

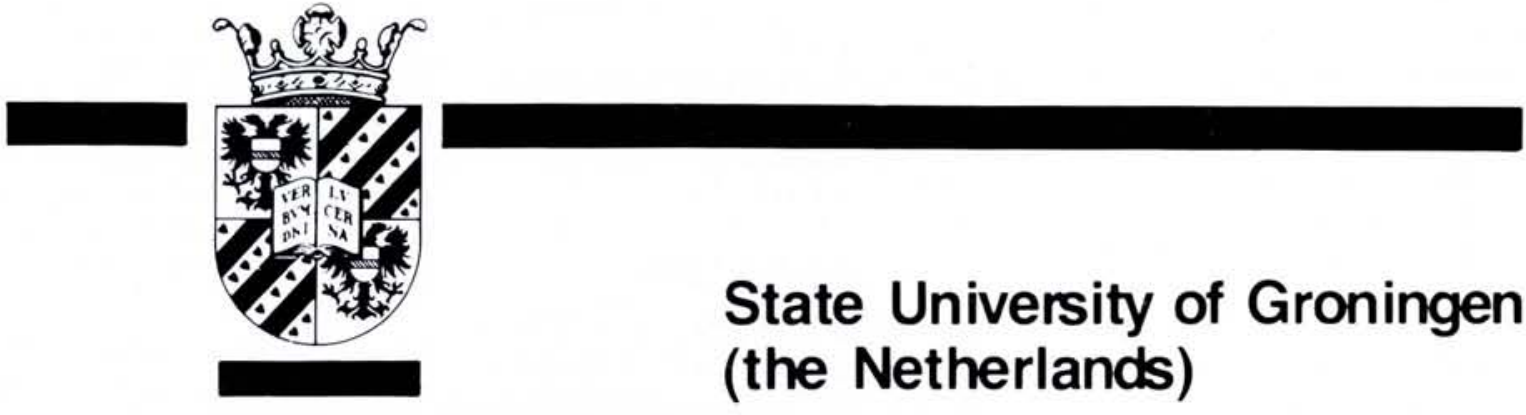

\section{professor of experimental physics}

(vac. nr. 810212/PhT)

Applications are invited by the Subfaculty of Physics of the University of Groningen, the Netherlands, for a Professor of Experimental Physics to be associated with the Kernfysisch Versneller Instituut (K.V.I.).

The Kernfysisch Versneller Instituut in Groningen is a national center for Nuclear Physics that is jointly sponsored by the University of Groningen and the national foundation for physics research, F.O.M. The regular staff of the K.V.I. consists of 75 persons, of which 35 are scientists, including both experimental and theoretical nuclear physicists.

The laboratory has a large $(K=160)$, variable energy cyclotron that can accelerate both light and heavy ions. With an axial injection system. that is under construction, and an ECRIS source it will be possible to accelerate totally stripped light ions up to oxygen, and heavier ions such as argon and krypton in a very high charge state.

Experimental facilities include a broad range Q3D magnetic spectrograph and excellent on- and off-line computing facilities. Close contacts are maintained with other physics institutes within and outside the Netherlands.

Applicants should have proven ability for original research in experimental nuclear physics with a broad interest and experience in nuclear structure and reaction mechanism studies. The successful applicant should be able to stimulate research.
$\mathrm{He}$ is expected to share in the scientific leadership of the Kernfysisch Versneller Instituut, and to be willing to take on responsabilities for the laboratory as a whole. Since the K.V.I. does not have rigid group structures, he should be willing to integrate himself into the laboratory and to work in a team. He is expected to share in teaching responsabilities of the department of Physics and to learn the Dutch language in a reasonable time.

The salary will be determined by age and experience and will be up to a maximum of $f$ 8773,- per month.

Applications should include a curriculum vitae and list of publications and should be sent to the Director of the Personnel Office, mentioning the vacancy number on both letter and envelope.

Director of the Personnel Office

Postbus 72

9700 AB Groningen

The Netherlands.

To arrive before June 1, 1981. Applicants should ensure that confidential reports in support of their application by three referees are sent directly to the Chairman of the selection committee Professor Siemssen and arrive before the closing date for applications. Further information about this position may be obtained from

Prof. Dr. R.H. Siemssen,

Zernikelaan 25,

9747 AA Groningen,

The Netherlands

tel.: 050-115724 
third and higher order, but the former errors may be eliminated easily by including the second term in (7) as a perturbation to first order.

\section{Muffin-tin Orbitals}

A convenient formalism in which the influences of potential and structure are explicitly separated is obtained when the primary set of basis functions (5) is chosen as so-called muffin-tin orbitals (MTOs). The muffin-tin orbitals used in connection with the atomic spheres approximation have tails decaying like $r^{-l-1} Y_{L}(\hat{f})$ (like potentials from static multipoles of order $\left.2^{l}\right)$ and they have expansions about other

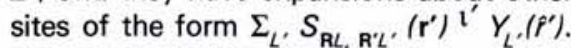
These tails thus have zero kinetic energy $\left(\nabla^{2} \chi=0\right)$ in the region between the atoms and this is the simplest and still reasonable approximation for the energy range of interest which is near the classical threshold for propagation. In this range the magnitude of the actual kinetic energy, $E-V$, is small compared with the centrifugal energy, $1(1+1) s^{-2}$, for the higher partial waves near the sphere boundaries. For the low partial waves it is sufficient to treat the spherical average of the interstitial region correctly, and this is done by the atomic spheres approximation.

The expansion coefficients, the so-called structure matrix, $S$, is proportional to

$$
\left|\mathbf{R}-\mathbf{R}^{\prime}\right|^{-\mathrm{l}^{-l^{\prime}-1} \mathrm{Y}_{\mathrm{t}}+\mathrm{l}^{\prime} \text {, } \mathrm{m}-\mathrm{m}\left(\mathbf{R}^{\prime}: \mathbf{R}^{\prime}\right)} \text {. }
$$

The potential parameters, derived from the values of the phi and phi-dot and their radial derivatives at the sphere, are expressed as $C_{R l}, \triangle_{R l}$ and $\gamma_{R l}$. Here, $C$ is the on-site energy of the muffin-tin orbital. The width parameter

$$
\triangle \equiv \frac{1}{2} s \phi^{2}(C, s) \equiv\left[\mu s^{2}\right]^{-1}
$$

is proportional to the probability-density at the sphere boundary, i.e. probability that the electron escapes from the atom, and it is inversely proportional to the intrinsic band mass, $\mu$, which is unity for free electrons. The third parameter, $\gamma$, is a measure of the slope of phi-dot at the sphere boundary (see Fig. 3) and, like the other two parameters, it depends only weakly on the choice of $E_{v}$.

With these muffin-tin orbitals it is now possible to set up the matching problem (3) in a simple way and the secular matrix becomes

$M(E)=\left[\triangle(E-C)^{-1}+\gamma+\ldots\right]^{-1}-S$

where the energy function in the square brackets depends only on the potential (this function is essentially the logarithmic derivative function of the atomic-sphere boundary) and the second term depends only on the structure, that is, on the positions of the surrounding spheres. It is then hardly surprising that the hamiltonian matrix in the representation of the nearly orthogonal orbitals (6) turns out to be

$H=C+\sqrt{ } \triangle S(1-\gamma S)^{-1} \sqrt{ } \triangle$.

In (8) and (9) all qualities are matrices in $\mathbf{R}$ and $L$, but the energy and the potential parameters are diagonal. The potential parameters $\gamma$ vary only little among related elements and we might therefore regard $\widetilde{S} \equiv S(1-\gamma S)^{-1}$ as a dressed structure matrix which, in contrast to the bare structure matrix, $S$, has short range.

Let us for simplicity consider an infinite crystal with just one atom per primitive cell. The potential parameters now depend only on $\mathrm{l}$ and the structure and hamiltonian matrices depend on $\mathbf{R}-\mathbf{R}^{\prime}$. The latter matrices may therefore be diagonalised by transformation from the site representation to the crystal-momentum, $\mathbf{k}$, or Blochvector representation, whereby the structure matrix, $S_{1 \partial, 1^{\prime} m^{\prime}}(\mathbf{k})$, is given by the lattice sums

$\sum_{\mathbf{R} \neq \dot{0} \exp (\boldsymbol{k} \cdot \mathbf{R})\left(s / R^{l+l^{\prime}+1}\right.} Y_{l+l}^{l}, m-m,(\hat{R})$.

These can be performed by hand when $\imath+\imath^{\prime} \geq 4$, while for lower $\mathrm{l}$-values they require the Ewald summation technique. The factor $s^{l+l^{\prime}+1}$ has been included in order that the structure matrix be independent of the lattice constant.

All the complicated $\mathbf{k}$-space dependence is thus contained in the structure matrix, and this may be calculated once and for all for a given crystal structure.

\section{APPLICATIONS}

The band structures of a large number of elemental metals $(\mathrm{K} \rightarrow \mathrm{Cu}, \mathrm{Rb} \rightarrow \mathrm{Ag}, \mathrm{Cs} \rightarrow$ $\mathrm{Au}$, La and $\mathrm{Ce}, \mathrm{Fr} \rightarrow \mathrm{Cf}$ ), the elemental semiconductors $(\mathrm{C}, \mathrm{Si}, \mathrm{Ge}$ ) and a number of binary transition metal and actinide compounds have been calculated self-consistently as a function of the lattice constant with the linear muffin-tin orbitals (LMTO) method in the atomic spheres approximation (ASA). In most cases the potential parameters and their volume derivatives have been tabulated. The calculated and observed equilibrium lattice constants for the $3 \mathrm{~d}$ - and $5 \mathrm{f}$-metals and for the $3 \mathrm{~d}$ monoxides are shown in Fig. 5. The increase of the lattice constants observed in the second half of the actinide and $3 \mathrm{~d}$ monoxide series is usually attributed to localisation of the $5 \mathrm{f}$ - and $3 \mathrm{~d}$-electrons respectively. This trend is seen to be well reproduced in the spin-unrestricted calculations.

Volume dependent properties are particularly simple to calculate and understand with the atomic-spheres approximation because only the potential parameters, not the k-dependent structure matrix, depend on the lattice constant. The electronic pressure, which is the derivative of the total electronic energy with respect to volume, can for instance be expressed analytically in terms of the potential parameters and the partial densities of one-electron states. This has been used throughout the above mentioned calculations.

The microscopic basis for Miedema's empirical theory of alloy formation, which was described in the September issue of

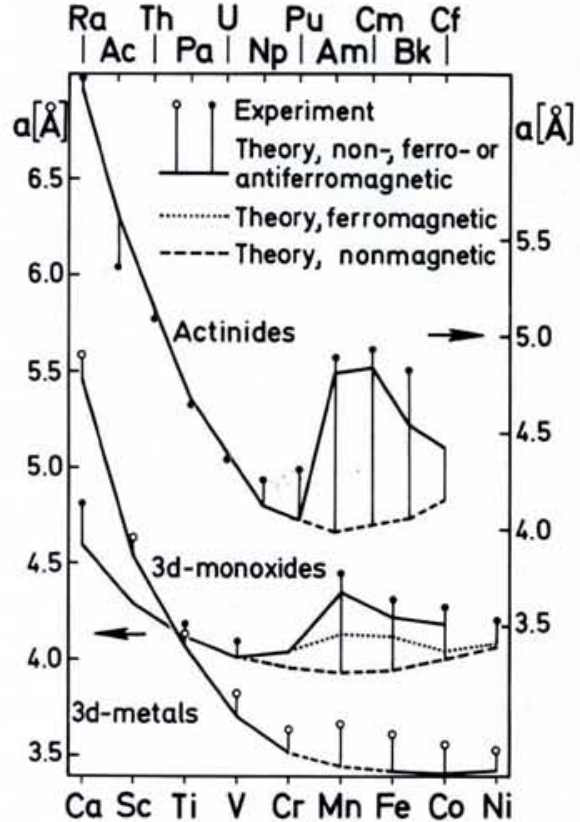

Fig. 5 - Comparison between experimental and theoretical lattice spacings, a, for the $3 d$ monoxides and the $3 d$ - and $5 f$-metals. For the monoxides of the $\mathrm{NaCl}$-structure, $a$ is the lattice constant, while for the elemental metals, having various closely packed structures, $a$ is the lattice constant of the face-centered-cubic structure with the proper atomic volume.

Europhysics News (1980), has been examined $^{2}$ ) by calculations for more than a hundred s-d, p-d and d-d bonded binary compounds using the socalled augmentedspherical-wave method, a direct descendant of the LMTO-ASA method. The conclusions, that the calculated heats of formation can be interpreted simply and that their accuracy is similar to that of Miedema's empirical results, give hope for the future use of band calculations in metallurgy.

Many other types of systems and problems have been approached using linear one-electron methods at various levels of approximation. In closing I might mention: Ground states and low-lying excited states of nearly all homonuclear diatomic molecules, magnetic moments near surfaces of transition metals, and chemical binding, magnetic and superconducting properties of ternary cluster compounds.

\section{REFERENCES}

1. Andersen O.K., Phys. Rev. B12 (1975) 3060.

2. Williams A.R., Gelatt C.D. and Moruzzi V.L., Phys. Rev. Lett. 44 (1980) 429 and Physics of Transition Metals Conf., Leeds, 1980.

Reviews:

3. Andersen O.K. and Jepsen O., Physica 91B (1977) 317.

4. Mackintosh A.R. and Andersen O.K. in Electrons at the Fermi Surface, M. Springford, Ed. (Cambridge University Press, 1980).

5. Andersen O.K., Skriver H.L., Nohl H. and Johansson B., Pure and Appl. Chem. 52 (1980) 93. 